\title{
A Quantitative Evaluation of Natural Language Question Interpretation for Question Answering Systems
}

\author{
Takuto Asakura $^{1(\mathbb{\triangle})}$, Jin-Dong Kim ${ }^{3}$, Yasunori Yamamoto ${ }^{3}$, Yuka Tateisi ${ }^{4}$, and \\ Toshihisa Takagi ${ }^{2}$ \\ 1 Department of Informatics, SOKENDAI, Tokyo, Japan \\ asakura@nii.ac.jp \\ 2 Department of Bioinformatics and Systems Biology, The University of Tokyo, Tokyo, Japan \\ tt@bs.s.u-tokyo.ac.jp \\ 3 Database Center for Life Science, Chiba, Japan \\ $\{j d k i m, y y\} @ d b c l s . r o i s . a c . j p$ \\ 4 National Bioscience Database Center, Tokyo, Japan \\ tateisi@biosciencedbc.jp
}

\begin{abstract}
Systematic benchmark evaluation plays an important role in the process of improving technologies for Question Answering (QA) systems. While currently there are a number of existing evaluation methods for natural language (NL) QA systems, most of them consider only the final answers, limiting their utility within a black box style evaluation. Herein, we propose a subdivided evaluation approach to enable finer-grained evaluation of QA systems, and present an evaluation tool which targets the NL question (NLQ) interpretation step, an initial step of a QA pipeline. The results of experiments using two public benchmark datasets suggest that we can get a deeper insight about the performance of a QA system using the proposed approach, which should provide a better guidance for improving the systems, than using black box style approaches.
\end{abstract}

\section{Introduction}

Recently, Linked Data (LD) has been recognized as an emerging standard for the integration of databases and the number of RDF Knowledge Bases (RKBs) is rapidly increasing $[4,16,27]$. While SPARQL is recognized as a standard tool for treating RKBs, authoring queries in SPARQL is not so easy especially for non-technicians [10]. For this reason, systems that allow users to search RKBs through NL questions (NLQs), the so-called Question Answering (QA) systems, are recognized as being highly useful. In particular, QA systems generating SPARQL queries from NLQs are called Semantic QA (SQA) systems (the exact definition is available in a study by Höffner [11]).

Benchmarking evaluations play an important role in improving SQA systems. While there are a number of existing evaluation methods, these methods essentially evaluate only the final answers per input NLQs [17]. However, since SQA systems have to involve various processes (e.g., parsing NLQs and finding URIs of entities), evaluations with only the final answers will not highlight the reasons why the results of the evaluations are unexpected. This limitation is considerably inconvenient for developers who are trying to improve their systems. 
With this observation in mind, we propose a new evaluation method for SQA systems with which we aim to provide subdivided evaluation results rather than checking only the final answers. One of the possible evaluation directions is to focus on how valid logical expressions can be generated from the input NLQs. In other words, evaluations on the NLQ interpreter, a module of SQA systems, are useful. As a similar attempt, Abacha et al. analyzed the false cases of their SQA system and classified them into (1) errors associated with the answer type and (2) errors associated with relation extraction [2]. Herein, we employ this approach to evaluate NLQ interpreters and present a calculation scheme for quantitative evaluation. The method has been implemented for evaluating a module of OKBQA, a highly generalized SQA framework (see Section 2.2), and it is available at https://gi thub.com/wtsnjp/eval_tgm. This program will be the first module of the subdivided evaluation framework for the entire SQA system.

\section{Benchmark Datasets and SQA Systems}

\subsection{Datasets}

There are several famous benchmarks for QA systems, e.g., WeBQuestions [3], SIMPLEQuestions [5] and BioASQ [1]. Although such datasets contain thousands of questionanswer pairs, which are also annotated with some other information, these are not suitable for our purpose because nothing that expresses the logical structures of the questions is contained in these datasets.

One of the formal languages or logical expressions is $\lambda$-Calculi, and FreE917 [6] has 917 pairs of NLQs and corresponding $\lambda$-Calculuses. However, using SPARQL as a logical expression is much more reasonable for our tasks. This is because questions that can be annotated with SPARQL clearly lie in the scope of the SQA systems, and both SPARQL queries or some similar expressions collected from datasets and those generated by SQA systems can be treated in exactly the same way (e.g., both SPARQL queries out of databases and generated SPARQL queries can be parsed by the same parser). For these reasons, two datasets comprising pairs of NLQs and SPARQL queries are chosen to be the benchmark datasets for our evaluation.

QALD As one of the most well-known evaluation tasks, QALD ${ }^{5}[17,7,23,24,22,26]$ contains a number of questions annotated with equivalent SPARQL queries (Table 1). Some of the datasets (e.g., those named multilingual) contain not only English questions but also questions in several other languages; however, we used only the English questions. The questions in the datasets that do not contain SPARQL queries (i.e., hybrid datasets from QALD-4-7) are annotated with pseudo queries instead. These are quite similar to SPARQL queries but can contain free text as the node of the triples, which makes the triple patterns of the pseudo queries different from those of the actual queries. Therefore, these datasets are inappropriate for our evaluation.

Practically, datacube from QALD-6 and largescale-test from QALD-7 are also inappropriate for our purpose. The SPARQL queries in the former datasets comprise a lot

\footnotetext{
${ }^{5}$ https://qald. sebastianwalter.org/
} 
Table 1. Overview of datasets provided by QALD. The rightmost column shows whether each dataset was used for our experiments (see Section 2.1 for detailed reasons).

\begin{tabular}{|c|c|c|c|c|c|}
\hline Challenge & Dataset & Size & Question (en) & SPARQL query & Used \\
\hline QALD-1 & dbpedia-\{train, test $\}$ & 100 & $\checkmark$ & $\checkmark$ & $\checkmark$ \\
\hline & musicbrainz-\{train,test $\}$ & 100 & $\checkmark$ & $\checkmark$ & $\checkmark$ \\
\hline QALD-2 & dbpedia-\{train,test $\}$ & 200 & $\checkmark$ & $\checkmark$ & $\checkmark$ \\
\hline & musicbrainz-\{train,test $\}$ & 200 & $\checkmark$ & $\checkmark$ & $\checkmark$ \\
\hline & participants-challenge & 7 & $\checkmark$ & $\checkmark$ & $\checkmark$ \\
\hline QALD-3 & esdbpedia-\{train,test $\}$ & 100 & $\bar{\checkmark}$ & $\bar{\checkmark}$ & $\bar{\checkmark}$ \\
\hline & dbpedia-\{train,test $\}$ & 199 & $\checkmark$ & $\checkmark$ & $\checkmark$ \\
\hline & musicbrainz-\{train, test $\}$ & 199 & $\checkmark$ & $\checkmark$ & $\checkmark$ \\
\hline QALD-4 & multilingual-\{train,test $\}$ & 250 & $\checkmark$ & $\checkmark$ & $\checkmark$ \\
\hline & biomedical-\{train,test\} & 50 & $\checkmark$ & $\checkmark$ & $\checkmark$ \\
\hline & hybrid-\{train,test $\}$ & 35 & $\checkmark$ & & \\
\hline QALD-5 & multilingual-\{train,test $\}$ & 350 & $\checkmark$ & $\checkmark$ & $\checkmark$ \\
\hline & hybrid-\{train,test $\}$ & 50 & $\checkmark$ & & \\
\hline QALD-6 & multilingual-\{train,test $\}$ & 450 & $\checkmark$ & $\checkmark$ & $\bar{\checkmark}$ \\
\hline & hybrid-\{train,test $\}$ & 75 & $\checkmark$ & & \\
\hline & datacube-\{train,test $\}$ & 150 & $\checkmark$ & $\checkmark$ & \\
\hline QALD-7 & multilingual-\{train, test & 314 & $\checkmark$ & $\checkmark$ & $\checkmark$ \\
\hline & hybrid-\{train,test $\}$ & 150 & $\checkmark$ & & \\
\hline & largescale-train & 100 & $\checkmark$ & $\checkmark$ & $\checkmark$ \\
\hline & largescale-test & $2 \mathrm{M}$ & $\checkmark$ & $\checkmark$ & \\
\hline & en-wikidata-\{train,test $\}$ & 150 & $\checkmark$ & $\checkmark$ & $\checkmark$ \\
\hline
\end{tabular}

of extended syntaxes; thus, it is difficult for us to treat them as valid SPARQL queries. The latter dataset has 2 million questions, but this dataset is mechanically generated by an algorithm using the questions available in the training dataset. Thus, largescale-test contains a large number of similar questions; hence, we have chosen to skip the dataset in our evaluation.

Moreover, although each newer dataset is not a proper superset of the dataset for the previous tasks, many questions appear multiple times throughout the datasets. Using the same questions more than once can cause bias in the evaluation results; hence, such occurrences should be avoided.

Due to these reasons, quite a few questions were discarded from our evaluations, but we could still obtain a reasonable number of questions annotated with appropriate SPARQL queries. The exact number of questions used for our experiments and some of the basic analyses conducted on them will be presented in Section 4.

LC-QuAD Largescale Complex Question Answering Dataset (LC-QuAD) ${ }^{6}$ [21] is a newer dataset that also contains 5,000 pairs of questions in English and SPARQL queries. This dataset is for machine learning-based QA approaches. It is also useful for our evaluation owing to its size and complexity. Unlike the largescale-test dataset from

${ }^{6}$ https://figshare.com/projects/LC-QuAD/21812 
QALD-7, LC-QuAD was carefully created to exclude questions that are similar to each other.

\subsection{The SQA Framework: OKBQA}

The Open Knowledge Base and Question-Answering (OKBQA) ${ }^{7}$ community has been developing the OKBQA framework by modularizing general SQA systems so that each module can be developed independently by experts in each of the related technologies $[13,12]$. Hence the framework share the same goal with our evaluation method. The main part of the framework or the SQA workflow comprises the following modules (Figure 1).

- Template Generation Modules (TGMs) take an NLQ $(q)$ as their input and return a list of SPARQL templates $\mathcal{T}(q)$, which are pairs of template queries and sets of slots $\left(\tau_{i}, S_{i}\right)$. Here, a template query, $\tau_{i}$, is similar to a SPARQL query, but all components of its triples are unbounded variables, and the set of slots $S_{i}$ holds the descriptions of the variables. Generally, SPARQL templates represent the semantic structures of the questions [25]. Therefore, typical TGMs create them by using some natural language processing (NLP) techniques.

- Disambiguation Modules (DMs) receive a SPARQL template $\left(\tau_{i}, S_{i}\right) \in \mathcal{T}(q)$ and identify resources corresponding to each of the slots in $S_{i}$. More specifically, a result of DMs $\mathcal{D}_{i}$ is a set of three tuples $\left(E_{j}, C_{j}, P_{j}\right)$, where each tuple is a list of slots to URIs mappings for entities, classes, and properties, respectively. Normally, DMs require RKB-dependent information in addition to their input from TGMs.

- Query Generation Modules (QGMs) generate actual SPARQLqueries $Q_{i, j}(q)$ based on a template $\left(\left(\tau_{i}, S_{i}\right) \in \mathcal{T}(q)\right)$ and three tuples of mappings $\left(E_{j}, C_{j}, P_{j}\right) \in \mathcal{D}_{i}(q)$. This module tends to generate many SPARQL queries for each input template.

- Answer Generation Modules (AGMs) query all specified RKBs using SPARQL queries generated by a QGM and return the list of final answers $\mathcal{A}(q)$ for the question $q$. The role of this module is not only collecting results from RKBs but also selecting and filtering the input SPARQL queries.

Because of this modular architecture, the users of the SQA system can freely choose the exact implementation to execute as each module in the SQA workflow, and for easing the collaboration, every module implementation has REST services to exchange their inputs/outputs. It is worth noting that the framework is particularly useful for our subdivided evaluations because it is helpful to clarify that the scopes of the evaluations and the evaluators developed for each module can be easily applied to multiple implementations.

Now, we can clearly declare the objective of this study, which is to define and develop an evaluation for the TGMs of the OKBQA framework. Currently, there are two TGM implementations for English QA: Rocknrole and LODQA. We evaluated both using our evaluation method.

\footnotetext{
${ }^{7}$ http://www. okbqa.org/
} 


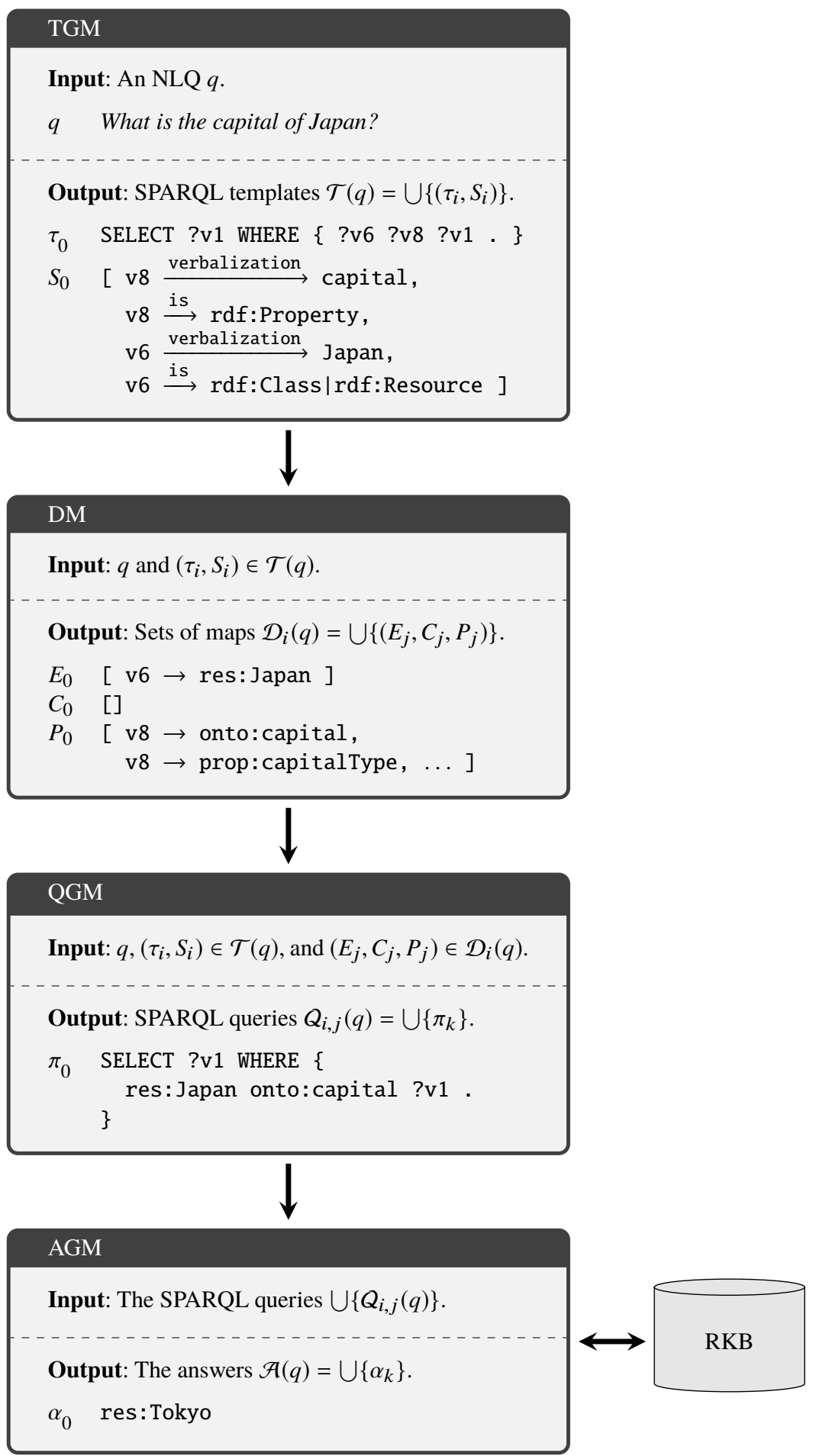

Fig. 1. The workflow of the OKBQA framework with the example outputs for the question "What is the capital of Japan?" The prefixes used in this figure are summarized in Table 3. Only the essences of the inputs or outputs of each module are shown here; hence, the actual APIs allow for attaching additional information to the inputs. For instance, many components of the outputs are annotated with a score so that AGMs will be able to select or filter the candidate SPARQL queries. 
Rocknrole Rocknrole ${ }^{8}$ [25] is a rule-based TGM implementation. The approach of this implementation is quite simple: first, the input question is parsed by the general natural language (NL) parser included in Stanford CoreNLP [18] and then converted to a SPARQL template query using predefined rules (e.g., the node who is renamed to AGENT). Because of its SPARQL templates generation scheme, the quality and coverage of the output are dependent on the rules.

LODQA Linked Open Data Question-Answering (LODQA) ${ }^{9}[14,8]$ is one of the SQA systems that generate SPARQL queries. LODQA has a modular architecture that resembles the OKBQA framework, and one of the modules of the system called Graphicator can be used as a TGM implementation solely by adjusting the output to conform to the TGM specification. The backend for deep relation extraction of the system is Enju [19], a stateof-the-art HPSG-based English parser. Then, a graph conversion algorithm involving tasks such as entity recognition and graph simplification is executed.

\section{Methods}

Figure 2 shows the overview of the calculation scheme in our evaluator. The detailed information about each process is described in this section.

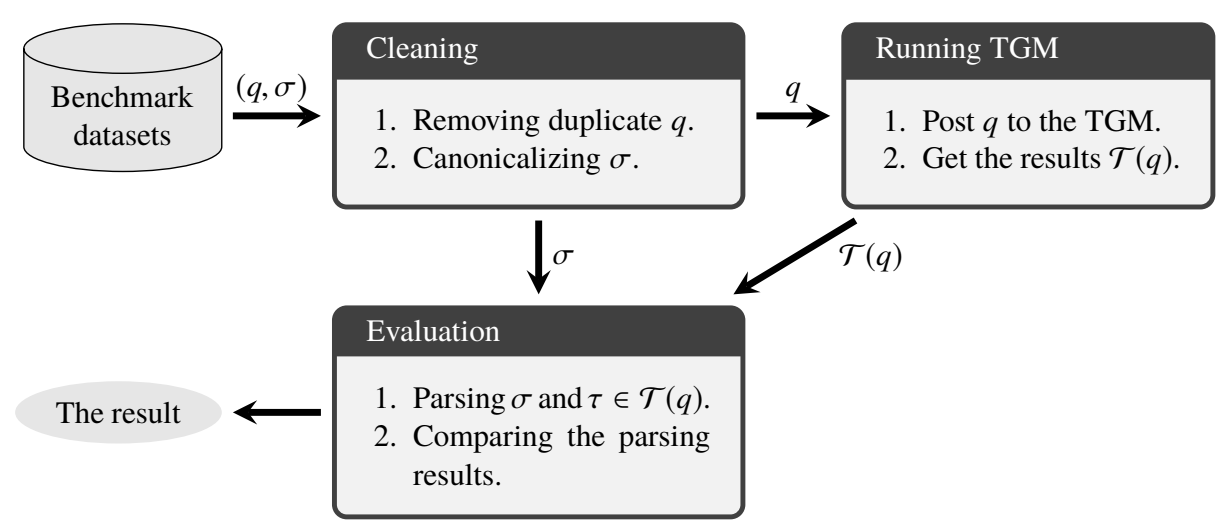

Fig. 2. The calculation scheme of the evaluation. The inputs of our evaluator are pairs of the NLQs and corresponding SPARQL queries $(q, \sigma)$. After executing all the processes, the evaluator outputs the evaluation results for the TGM.

\footnotetext{
${ }^{8}$ http://repository.okbqa.org/components/21

${ }^{9}$ http://lodqa.org/
} 


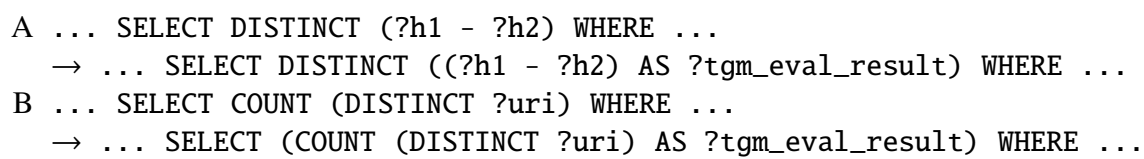

Fig. 3. Examples of the substitutions to modify the SPARQL queries containing extended syntaxes. Here, the names of variables added by our scripts have prefix tgm_eval_ to avoid any name confliction.

Table 2. The REST services of the TGMs. When users send POST requests on HTTP, the services will run the TGM internally and return the results to users. While Rocknrole supports English and Korean, LODQA currently supports only English.

TGM name Service URL

Languages

Rocknrole http://ws.okbqa.org:1515/templategeneration/rocknrole en, ko

LODQA http://lodqa.org/template.json

\subsection{Preparation}

Before using the pairs of the NLQs and SPARQL queries from the datasets of QALD and LC-QuAD, two simple processes were required to be applied to these queries for cleaning purposes. First, due to the existence of duplicate questions in both datasets, it was necessary to remove those duplicate questions from the input of the evaluator. Duplicate NLQs that were paired with different SPARQL queries existed in the QALD datasets, and thus, the queries from the newer datasets may be more effective compared with those contained in the older ones. Consequently, the pairs from the newer datasets were selected in such cases. Second, the SPARQL queries from both datasets included extended syntaxes of Virtuoso, ${ }^{10}$ so many of these queries did not satisfy the specification of SPARQL 1.1, ${ }^{11}$ which may cause parse failure of the queries. To avoid such trivial errors, these invalid queries were modified to conform to the official specification before the evaluation. This process was performed in an ad-hoc manner: we made the modification using regular expressions in our formatter scripts to create the input data files (Figure 3 ).

After filtering and modification, the remaining NLQs constituted our benchmark datasets. These datasets were then fed to the two TGMs and the outputs were retrieved through their REST services (Table 2). As a result, we obtained the pairs of NLQs and corresponding SPARQL queries $(q, \sigma)$ from the datasets as well as the pairs of template queries and sets of slots $\mathcal{T}(q)=(\tau, S)$ from the two TGMs. As mentioned in Section 2.2, the specification of the OKBQA framework allows TGMs to output multiple SPARQL templates for an NLQ, but both Rocknrole and LODQA currently output at most one template. Hence, we did not consider the cases of multiple TGM outputs from an input for our experiments.

As the last step for our preparation, we input both the SPARQL queries $\sigma$ from the datasets and template queries $\tau$ from the TGMs outputs into a SPARQL parser.

\footnotetext{
10 https://virtuoso.openlinksw.com/

11 https://www.w3 .org/TR/sparql11-query/
} 
Table 3. The namespace mappings used to initialized the internal SPARQL parser of RDFLib in our experiments. Since our evaluation for TGMs does not check any particular resource, the exact URIs shown here is not so important.

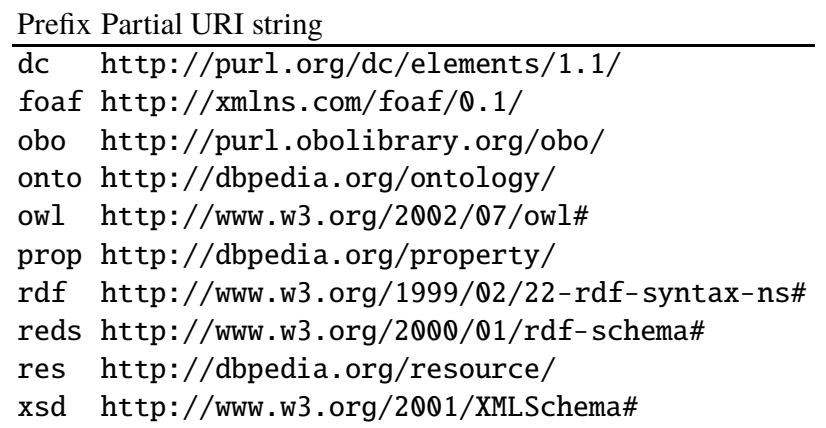

Specifically, we used an internal SPARQL parser from RDFLib ${ }^{12}$ solely because it is convenient and fast to call this parser from our evaluation scripts written in Python. The parser outputs the internal expressions of the parsed SPARQL queries, similar to SPARQL syntax expressions or SPARQL Algebra, and we can easily extract the logical structure of the SPARQL queries, e.g., the triple patterns and the length of the answers from such queries. Technically, the parser must be initialized with a few namespace mappings (Table 3) because some of the SPARQL queries in the datasets do not have an explicit declaration of the prefixes.

\subsection{Evaluation}

The goal of our evaluation is to judge the qualities of the outputs of TGMs independently from the other part of the SQA systems (namely, DMs, QGMs, and AGMs). For this reason, we leave the analyses on the sets of slots $S$ and comparing graph similarity to another step, which will follow the TGM evaluation (see Section 5.3). Thus, our method focus on foundational analyses particularly on the template queries $\tau$.

To achieve our goal for subdivided evaluation, we established six evaluation criteria based on three aspects: (1) robustness of a TGM, (2) validity of query types and the ranges (i.e., lengths and offsets) expressed in template queries, and (3) accuracy of the graph patterns in template queries. For each aspect, two actual evaluation criteria have been developed, as listed in Table 4. Our evaluator is implemented to check every output of a TGM via a comparison with the corresponding queries from the benchmark dataset to verify whether any of the six criteria are met. If an output clears all the criteria, it is determined to be good.

For the convenience of the developers of TGMs, we also categorized the criteria into two severity levels: critical and notice. The difference between the two levels is related to the impact on the general evaluation criteria, e.g., recall and precision, which are

\footnotetext{
12 https://rdflib.readthedocs.io/en/stable/
} 
Table 4. Overview of the evaluation criteria. Our evaluator checks every output of a TGM to evaluate whether the output has any problem when compared with each criterion in the exact order shown here. If errors are found, the error that is found first is considered. For details of each criteria, see Section 3.2-3.2.

\begin{tabular}{lll} 
No. Evaluation criteria & Aspects & Level \\
\hline 1 TGM failure & Robustness & Critical \\
2 Syntax & Robustness & Critical \\
3 Question type & Query types and ranges Critical \\
4 Disconnected target & Graph patterns & Critical \\
5 Wrong range & Query types and ranges Notice \\
6 Disconnected triple & Graph patterns & Notice
\end{tabular}

widely used for evaluation in information systems, e.g., QALD campaign [17]:

$$
\begin{aligned}
\operatorname{Recall}(q) & =\frac{\text { Number of correct system answers for } q}{\text { Number of gold standard answers for } q}, \\
\operatorname{Precision}(q) & =\frac{\text { Number of correct system answers for } q}{\text { Number of system answers for } q} .
\end{aligned}
$$

For instance, if a SPARQL template, $\mathcal{T}(q)$, is judged to have a critical error, it means there is no chance of a correct answer, regardless of the performance of other modules. Additionally, the contribution of the template for precision and recall will be zero. On the contrary, if a $\mathcal{T}(q)$ is determined to have a notice problem, it means there is still a chance for obtaining correct answers, irrespective of how low this chance is. Thus, its contribution to precision and recall may not be zero.

Robustness The first two steps for our evaluation are related to the robustness of the TGMs. We call it a TGM failure error if the status code of the HTTP response from the REST service is not 200 , which means that somehow the TGM did not return normal results (e.g., a kind of internal error was raised for the input). If the REST service would have returned a SPARQL template, $\mathcal{T}(q)$, the parsing result of the template query $\tau$ would have been checked for the next step. As explained in Section 2.2, a valid template query is also valid as a SPARQL query. Therefore, a template query for which the parsing result is "syntax error" has syntactic problems. Since both the problems concerning the criteria explained here will make it difficult to follow the steps of the framework, these problems are classified into critical errors.

Query Types and Ranges Generally, NLQs, which can be treated as the inputs of SQA systems, are roughly categorized into the following question types [11,1].

- Yes/no questions are questions that can be answered simply as "yes" or "no" (e.g., "Are there drugs that target the Protein kinase $\mathrm{C} \beta$ type?"). These questions can be converted directly to SPARQL queries using the ASK form, i.e., the so-called ask queries. 
- Factoid questions require one or more entities as their answers (e.g., "Which drugs have no side-effects?"). The aim of these questions can be easily reached by the most common SPARQL queries using the SELECT form, namely select queries. Sometimes, the questions that require more than one answer are distinguished from this category (often referred to as "list questions"), but we did not separate that category from factoid questions because it is a trivial matter for SPARQL queries.

- Summary questions are questions that are not categorized into any of the previous types (e.g., "Why do people fall in love?"). The questions typically require text as the answers; therefore, the questions belonging to this class are out of the scope of SPARQL queries.

In summary, an input NLQ of an SQA system is basically classified into yes/no questions or factoid questions, which can be easily detected by checking the type of the annotated SPARQL query. Using this idea, we determined whether TGMs can accurately recognize the question types by comparing the parsing results of the dataset SPARQL queries $\sigma$ and the template queries $\tau$ : in the case wherein one of the queries is an ask query and the other is not and vice versa, we judged that the TGM failed to recognize the question type of the NLQ. This error is critical because incorrect types of queries always return the wrong type of answers.

Focusing on the factoid questions, it is worth considering more detailed classification among them. There are some questions, e.g., "Who are the four youngest MVP basketball players?" wherein the number of answers have important meanings. Moreover, the positions or the offsets of the answers (i.e., the positions in the sorted lists of answer candidates) are important in some questions, e.g., a SPARQL query corresponding to a question such as "What is the largest country in the world?" should consider the first entity from the (sorted) candidate entities while it is desirable for a SPARQL query to consider the second question "What is the second highest mountain on Earth?" Herein, we refer to these questions as range-specified factoid questions. The ranges of the answers, a pair of length $l$ and starting position $s$, can be expressed in a SPARQL query by adding clauses, such as "LIMIT $l$ OFFSET $(s-1)$." Thus, we checked every template query $\tau$ that correctly recognized the original question as a factoid question (if and if only one of the queries $\sigma$ and $\tau$ was not an ask query) and had the appropriate range specification in the query again using the parsing result of both the $\sigma$ and $\tau$ queries. If a range $(l, s)$ explicitly appeared in the SPARQL query $\sigma$ and either one of the lengths and starting positions in the template query $\tau$ were different from $l$ and $s$, respectively, the template was judged to have wrong range for the answers. Since adding the range annotations to template queries is an optional behavior of TGM to increase the precision, this criterion is rightfully categorized to notice.

Graph Patterns The basic structures of SPARQL queries (select queries in a precise sense) can briefly be expressed as follows [15].

SELECT 〈result description〉 WHERE 〈graph patterns〉,

where the part $\langle$ graph patterns $\rangle$ is a set of triple patterns and $\langle$ result description $\rangle$ is an enumeration of the variables requested to be solved by the queried RDF store, possibly with some arithmetic operators. Herein, we simply call these variables "target variables." 


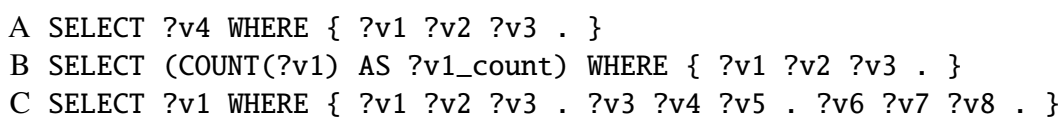

Fig. 4. Examples of template queries. A. The target variable ?v4 is a disconnected target because it does not appear in the graph patterns. B. The target variable ?v1_count does not appear in the graph patterns, but the variable is bound as the number of ?v1 and ?v1 appears in the patterns "?v1 ?v2 ?v3." Therefore, this template query does not have any problem. C. This query has a disconnected triple: while the first two triples in the graph patterns have a connection to the target variable ?v1, the last triple does not.

For each template query that has the form of select queries, we checked whether all target variables appeared in the $\langle$ graph patterns $\rangle$. If there were target variables that did not exist in the $\langle$ graph patterns $\rangle$, then an alert was generated as a disconnected target error (Figure 4A, 4B). This is one of the critical errors because the queries having this problem will retrieve nothing for those targets.

The last criterion of our evaluation is related to another kind of analysis on $\langle$ graph patterns $\rangle$. If there are triple patterns that are disconnected from any target, this can be a cause of reducing the meaningful results from the final answers for nothing. Thus, our evaluator found template queries having those triples that were highly unnecessary, which were considered as disconnected triple notifications (Figure 4C).

\section{Results}

After removing the duplicate NLQs from the datasets, we obtained 1,011 pairs of NLQs and SPARQL queries from the QALD datasets and 4,977 pairs from LC-QuAD (Figure 5). For the datasets pertaining to QALD and LC-QuAD, the ratio of yes/no questions was $8.4 \%$ and $7.4 \%$, respectively. In contrast, $7.0 \%$ of the factoid questions from QALD are range-specified, which are useful to check the existence of the wrong range criterion, but there are no range-specified factoid questions in LC-QuAD. All pairs were entered into our evaluator, and every NLQ in them was successfully sent to the REST services of the TGMs. Likewise, every query in the pairs was parsed by the SPARQL parser in RDFLib without any issues owing to the normalization adopted in our formatter.

Table 5 summarizes the problems of TGM outputs we found through evaluation using the whole datasets (5,988 questions in total). These problems are classified into the six criteria, as defined earlier. Overall, $9.9 \%$ of the SPARQL templates produced by Rocknrole had critical errors and $48.7 \%$ of these were alerted to have notice problems. Similarly, $7.8 \%$ of the templates generated by LODQA had critical errors and $1.1 \%$ of these were alerted to have notice problems (Figure 6).

\section{Discussions}

\subsection{Qualitative Evaluation of the TGMs}

Rocknrole Since Rocknrole is a rule-based NLQ interpreter, the coverage of the system is dependent on the rules. According to our evaluation results, we determined that 


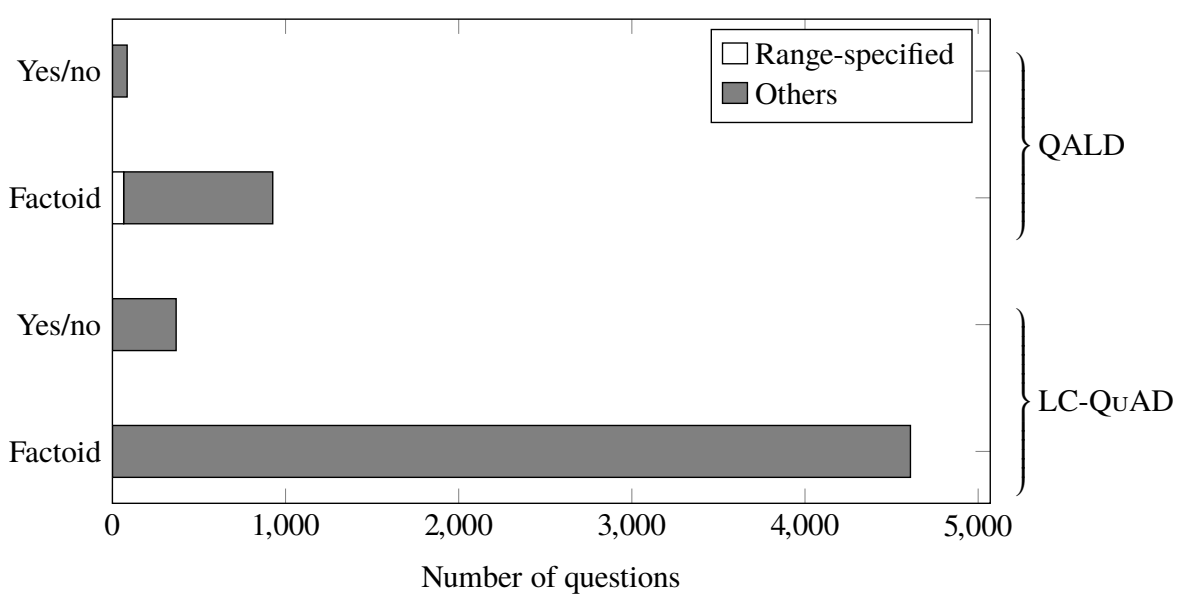

Fig. 5. Sizes of the datasets used in our experiments. The datasets from QALD contain 85 yes/no questions and 926 factoid questions, 65 of which were range-specified. On the contrary, the dataset from LC-QuAD comprises 368 yes/no questions and 4,609 factoid questions, none of which had a range specification.

Table 5. Number of the problematic SPARQL templates from the two TGMs for each of the evaluation criteria (DC represents the term "disconnected").

TGM name TGM failure Syntax Question type DC target Wrong range DC triple

\begin{tabular}{lrrrrrr}
\hline Rocknrole & 0 & 0 & 262 & 330 & 28 & 2,898 \\
LODQA & 1 & 18 & 446 & 0 & 64 & 0
\end{tabular}

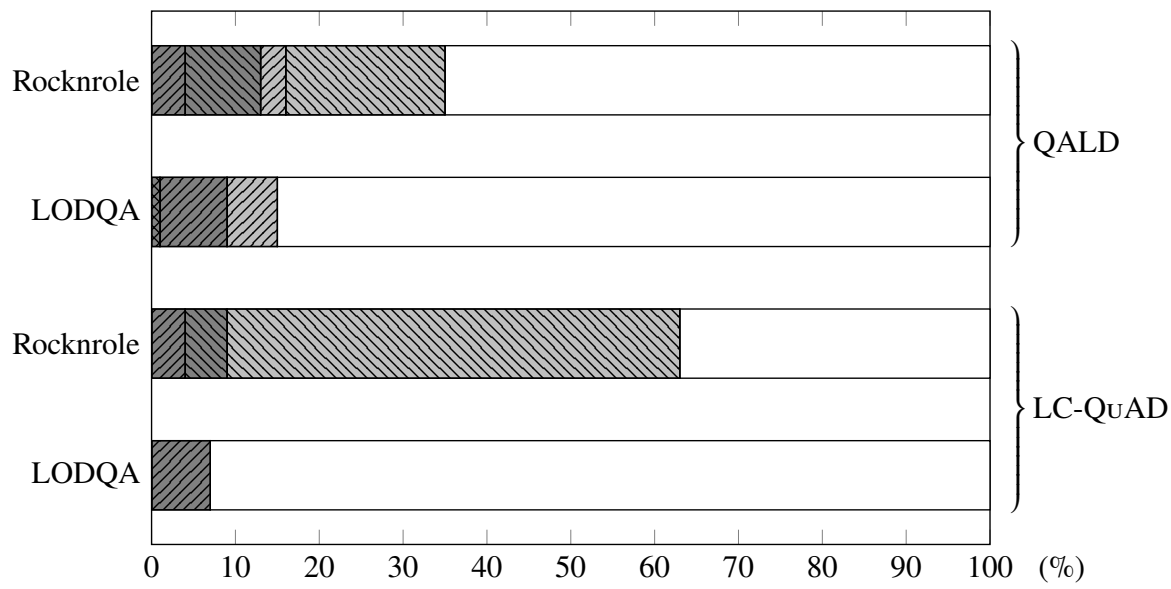

$\square$ TGM failure $\mathbb{X}$ Syntax
$\square \Delta$ Wrong range $\mathbb{Q}$ Disconnected triple $\square$ Good

Fig. 6. Ratios of the problematic SPARQL templates from the two TGMs for all evaluation criteria. The dark segments $\square$ represent the ratios for the critical criteria, and the lighter segments $\square$ show those for the notice criteria. 
(1) this TGM covers both the question types, i.e., yes/no questions and factoid questions, which are possibly assigned to SQA systems, (2) the system is also able to add range specification to the template queries, and (3) the system, however, often fails to generate good SPARQL templates. It is worth noting that Rocknrole can perfectly recognize yes/no questions in our experiments, but it judged $4.7 \%$ of the factoid questions as yes/no questions throughout all the datasets used herein. In addition, the TGM failed to add appropriate range specification to its outputs for nearly half of the range-specified factoid questions $(45.2 \%)$ in the QALD datasets. Note that this level of insight into the performance of the TGM is something that could only be achieved through the subdivided evaluation proposed in this study.

One possible way to improve Rocknrole to prevent the occurrence of errors, particularly the question-type, disconnected target, and wrong range errors, is to add predefined rules to decrease the error rates. For this purpose, the false cases dumped by our evaluator will be useful. Furthermore, Rocknrole can only enhance the system to remove disconnected triples so that such triples will not decrease the values of recalls.

LODQA As for the TGM provided by LODQA, the evaluation shows that (1) it does not distinguish the yes/no- and factoid-type questions, (2) it does not produce range specification to the template queries, and (3) it, however, produces good SPARQL templates more stably than Rocknrole. Since the system does not cover yes/no questions and range-specified factoid questions, the number of question-type and wrong range problems presented in Table 5 and Figure 6 are only those problems that are included in the datasets.

\subsection{Quality of the Datasets}

Figure 5 shows that the questions in the QALD datasets are richer in diversity than those in LC-QuAD. In fact, LC-QuAD is not sufficiently diverse to be used solely for our evaluation because it contains no range-specified factoid questions.

The size of both the datasets seems sufficiently large for our evaluation task because we were able to find at least one case for each of the criteria. Nevertheless, the larger the size is, the better the dataset for our evaluation. In this regard, LC-QuAD has an advantage. The generation process of LC-QuAD is quite unique: the NLQ in the dataset is transformed from SPARQL queries, which is completely opposite to the generation processes of the other well-known datasets, including QALD [21]. As the translations from SPARQL queries to NLQs were conducted using specific question templates called "normalized natural question templates," if the variety of the templates is increased, the dataset will be more useful for our evaluation.

\subsection{Unsuitable evaluation criteria}

Several methods have been proposed to determine the similarity degrees or distances among SPARQL queries [15,9], which seems to be useful for evaluating SQA systems. Although this may also be true for evaluating the whole OKBQA framework, these methods are not appropriate for our evaluation because our evaluation currently focuses on only 
TGMs. First, most aforementioned measurements cannot be applied to SPARQL queries that do not contain URIs. Second, comparing or measuring the similarity between the graph patterns of the SPARQL queries without resource annotations seems meaningless because one semantic structure of a question can generally be expressed in several forms of SPARQL queries.

Another possible criterion is the expected type of the answer, namely the type or class of the target variable in the template queries. However, this is also not appropriate for our evaluation because template queries do not contain any URI. We thus reserve this for the evaluation of other modules, which follow a TGM in a QA workflow.

\subsection{Limitations, possible extensions, and future work}

Both TGM implementations currently do not generate more than one SPARQL template for an NLQ, whereas the specification of the OKBQA framework allows TGMs to generate multiple templates for an input. For this reason, currently, we simply took the first template from the result lists, the length of which is always one, returned by TGMs; however, this behavior is required to be changed for TGMs that generate more than one templates for an NLQ. We think we can always take standard approaches which are broadly understood. For example, we can count positives/negatives and evaluate the performance in terms of precision and recall.

Though we evaluated only two TGMs for our experiments, our methods and implementation can be easily applied to other TGM implementations by merely specifying the URL of the REST service. Other datasets from QALD and LC-QuAD can also be used for the evaluation. Our evaluation is not specialized for English questions; thus, if applicable TGMs and datasets are provided, these TGMs can also be tested for other languages. Furthermore, our evaluation methods can possibly be applied not only to TGM in the OKBQA framework but also to every SQA system that generates SPARQL queries because the methods are solely based on the results of a general SPARQL parser. The results in such cases will demonstrate the performance of the functions corresponding to the TGM of the systems, which will be helpful in improving the systems as well.

Finally, for future research, defining and developing similar subdivided, systematic, and semantic evaluations of the other modules of the OKBQA framework will also be interesting. Evaluation methods optimized for more complex questions than our current corpus, such as those discussed by Talmor et al. [20], should also be implemented. Simultaneously, these evaluations will be helpful in improving the ability of the entire SQA system.

\section{Conclusions}

Herein, we proposed a systematic semantic evaluation for TGMs, which are a type of subdivided modules of SQA systems. Our evaluation results for the two publicly available TGMs revealed that in comparison with existing methods, the new evaluation method can extract and provide much more detailed information on their performance. Specifically, the limitations and the problems of the TGMs were detected; these are hoped to be fixed in the future. The information from our evaluator will be useful for addressing this issue. 
Improvement of SQA systems is important for expanding the use of LD, and this paper showed that the presented evaluation method has a good potential to play an important role for advancing the technology. Therefore, it will be worth to extend the approach to other modules of SQA systems, and even to other SQA frameworks, which is remained as a prospective future work.

\section{References}

1. Balikas, G., Krithara, A., Partalas, I., and Paliouras, G.: BioASQ: A Challenge on LargeScale Biomedical Semantic Indexing and Question Answering. In: Multimodal Retrieval in the Medical Domain, pp. 26-39 (2015)

2. Ben Abacha, A., and Zweigenbaum, P.: Medical Question Answering: Translating Medical Questions into Sparql Queries. In: Proceedings of the 2nd ACM SIGHIT International Health Informatics Symposium, pp. 41-50 (2012)

3. Berant, J., Chou, A., Frostig, R., and Liang, P.: Semantic Parsing on Freebase from QuestionAnswer Pairs. In: Proceedings of EMNLP, pp. 1533-1544 (2013)

4. Bizer, C., Heath, T., and Berners-Lee, T.: Linked DataâĂŤThe Story So Far. International Journal on Semantic Web and Information Systems 5(3), 1-22 (2009)

5. Bordes, A., Usunier, N., Chopra, S., and Weston, J.: LC-QuAD: A Corpus for Complex Question Answering over Knowledge Graphs. In: International Semantic Web Conference 2017, pp. 210-218

6. Cai, Q., and Yates, A.: Large-scale Semantic Parsing via Schema Matching and Lexicon Extension. In: Proceedings of the 51st Annual Meeting of the Association for Computational Linguistics (Volume 1: Long Papers), pp. 423-433 (2013)

7. Cimiano, P.: Multilingual Question Answering over Linked Data (QALD-3): Lab Overview. In: Information Access Evaluation. Multilinguality, Multimodality, and Visualization: 4th International Conference of the CLEF Initiative. Pp. 321-332 (2013)

8. Cohen, K.B., and Kim,J.-D.:Evaluation of SPARQL query generation from natural language questions. In: Proceedings of the Joint Workshop on NLP\&LOD and SWAIE, pp. 3-7 (2013)

9. Dividino, R.Q., and Gröner, G.: Which of the following SPARQL Queries are Similar? Why? In: Proceedings of the First International Conference on Linked Data for Information Extraction, pp. 2-13 (2013)

10. Harris, S., Seaborne, A., and Prudhommeaux, E.: SPARQL 1.1 query language. W3C recommendation 21(10) (2013)

11. Höffner, K.: Survey on challenges of question answering in the semantic web. Semantic Web 8(6), 895-920 (2017)

12. Kim, J.-D.: OKBQA Framework towards an open collaboration for development of natural language question-answering systems over knowledge bases. In: International Semantic Web Conference 2017

13. Kim, J.-D., Choi, G., Kim, J.-U., Kim, E.-K., and Choi, K.-S.: The Open Framework for Developing Knowledge Base And Question Answering System. In: Proceedings of the 26th International Conference on Computational Linguistics, pp. 161-165 (2016)

14. Kim, J.-D., and Cohen, K.B.: Natural language query processing for SPARQL generation: A prototype system for SNOMED CT. In: Proceedings of BioLINK SIG 2013, pp. 32-38

15. Le, W., Kementsietsidis, A., Duan, S., and Li, F.: Scalable multi-query optimization for SPARQL. In: Data Engineering (ICDE), 2012 IEEE 28th International Conference on, pp. 666-677 (2012)

16. Lehmann, J.: DBpedia-a large-scale, multilingual knowledge base extracted from Wikipedia. Semantic Web 6(2), 167-195 (2015) 
17. Lopez, V., Unger, C., Cimiano, P., and Motta, E.: Evaluating question answering over linked data. Web Semantics: Science, Services and Agents on the World Wide Web 21, 3-13 (2013)

18. Manning, C.D.: The stanford corenlp natural language processing toolkit. In: Proceedings of 52nd Annual Meeting of the Association for Computational Linguistics: System Demonstrations, pp. 55-60 (2014)

19. Miyao, Y., and Tsujii, J.: Feature forest models for probabilistic HPSG parsing. Computational linguistics 34(1), 35-80 (2008)

20. Talmor, A., and Berant, J.: The Web as a Knowledge-base for Answering Complex Questions. In: Proceedings of the 2018 Conference of the North American Chapter of the Association for Computational Linguistics: Human Language Technologies, Volume 1, pp. 641-651 (2018)

21. Trivedi, P., Maheshwari, G., Dubey, M., and Lehmann, J.: Lc-quad: A corpus for complex question answering over knowledge graphs. In: International Semantic Web Conference 2017, pp. 210-218

22. Unger, C., Ngomo, A.-C.N., and Cabrio, E.: 6th Open Challenge on Question Answering over Linked Data (QALD-6). In: Semantic Web Challenges: Third SemWebEval Challenge at ESWC 2016, pp. 171-177

23. Unger, C.: Question Answering over Linked Data (QALD-4). In: Working Notes for CLEF 2014 Conference, pp. 1172-1180

24. Unger, C.: Question Answering over Linked Data (QALD-5). In: Working Notes of CLEF 2015 - Conference and Labs of the Evaluation forum

25. Unger, C.: Template-based question answering over RDF data. In: Proceedings of the $21 \mathrm{st}$ international conference on World Wide Web, pp. 639-648 (2012)

26. Usbeck, R.: 7th Open Challenge on Question Answering over Linked Data(QALD-7). In: Semantic Web Challenges: 4th SemWebEval Challenge at ESWC 2017, pp. 59-69

27. Vrandei, D.: Wikidata: a new platform for collaborative data collection. In: Proceedings of the 21st International Conference on World Wide Web, pp. 1063-1064 (2012) 